\title{
PHASE SEPARATIONS, LIQUID CRYSTAL ORDERING AND MOLECULAR PARTITIONING IN MIXTURES OF PEG AND DNA OLIGOMERS.
}

\author{
Simone Di Leo ${ }^{1}$, Marco Todisco ${ }^{1}$, Tommaso Bellini ${ }^{1}$ and Tommaso P. Fraccia ${ }^{1,2}$ \\ 1 - Dipartimento di Biotecnologie Mediche e Medicina Traslazionale, Università di Milano, via Fratelli \\ Cervi 93, I-20090 Segrate (MI), Italy \\ 2 - Dipartimento di Scienze Umane e Promozione della Qualità della Vita, Università San Raffaele di Roma, \\ via di Val Cannuta, 247, I-00166 Roma (RM), Italy
}

Liquid crystals ordering of DNA and RNA oligomers relies on the presence of inter-duplex end-to-end attraction, driving the formation of linear aggregates. Such interactions are gauged, at a macroscopic level, by the osmotic pressure at the isotropic-nematic and nematic-columnar phase transitions. We studied aqueous solutions of PEG and DNA duplex-forming oligomers, finding that there is a wide range of concentrations in which these mixtures phase separate into coexisting PEG-rich and DNA-rich phases, the latter being either in the isotropic state or ordered as a nematic or columnar liquid crystal. We determined the phase diagram in mixtures of PEG and DNA duplexes with different terminal motifs - blunt ends, sticky overhangs, aggregation-preventing overhangs - and measured the partitioning of the species in the coexisting phases. On this basis, we determined the osmotic pressure as a function of the DNA concentration across the phase diagram. We compared the equation of state obtained in this way with both the CarnahanStarling equation of state for hard spheres and with the pressure predicted by computer simulations of a system of aggregating cylinders. We obtain a good agreement between experiments and simulations, and end-to-end attraction energies of the order of $6 \mathrm{kcal} / \mathrm{mol}$, a bit larger than expected, but still in agreement with the current models for DNA-DNA interactions.

KEYWORDS:

liquid crystals, DNA self-assembly, PEG-DNA phase separation, aqueous two-phase systems, equation of state, DNA osmotic pressure 


\section{INTRODUCTION}

Concentrated aqueous solutions of DNA oligomers paired in duplexes have been reported to order into Liquid Crystal (LC) phases [1-4]. This tendency toward LC ordering is quite robust, being found in a variety of systems, ranging from well-paired ("blunt ended") duplexes [1], to duplexes with mutually interacting ("sticky") overhangs [2], to duplexes including various degrees of defects, to structures alternating double and single strands [5], to fully random sequences [6]. LC have been found in longer (up to 20 bases) and shorter (down to 4 bases) DNA sequences [3,4]. Recently, LC have been observed even in solutions of single nucleobases [7], a finding that demonstrates that stacking attractive forces [8] are the main driving force of LC ordering. Despite being at the core of DNA stability, stacking forces are still a matter of investigation [9], since both their fundamental nature and their strength are not fully understood. In particular, stacking forces between independent duplexes have been quantified only via atomistic simulations [10].

The role of end-to-end attractions in the LC ordering of DNA duplexes has been studied via coarsegrained models. Specifically, LC ordering has been predicted in suspension of cylindrical monomer having steric repulsion and attractive interactions between their terminal bases only, which were investigated via computer simulations [11-14]. This is summarized in Fig. 1 in which we plot the volume fraction $(\phi)$ pressure (P) equation of state obtained from Monte Carlo simulations presented in Ref. [11] for a system of hard cylinders $(\mathrm{L} / \mathrm{D}=2)$, with various choices of base-to-base interaction strength $\mathrm{E}, 0<\mathrm{E}<12 \mathrm{k}_{\mathrm{B}} \mathrm{T}$. While at low pressure the system is an isotropic fluid (ISO), as concentration and pressure rise it may develop nematic LC $(\mathrm{N})$, columnar LC (COL) and columnar crystal $(\mathrm{C} 2)$ phases, depending on the interaction strength. As expected, the pressure at which the ISO-LC transition occurs decreases with increasing interaction strength. Thus, the measure of the pressure of the phase transition, which in the experimental systems would be the osmotic pressure ( $\Pi$ ) of the DNA solution, enables estimating the strength of the intercylinder attractive interactions. Evaluations of DNA inter-duplex interactions were elaborated before by a quantitative analysis of the ISO-LC phase boundary $[3,4,7]$. The measure of the pressure would enable a different, and possibly more direct route to this quantity. In Fig. 1 we also plot, as a simpler references: $\Pi_{\mathrm{CS}}(\phi)$, the Carnahan-Starling (CS) equation of state for hard spheres [15], which lays close to the ISO branches of the simulation (black line), and the equation of state of the ideal gas (dashed line).

A simple strategy to measure the osmotic pressure of DNA oligomers in solution is to exploit the phase separation that is observed when the flexible hydrophilic polymer polyethylene glycol (PEG) is solubilized in the same sample. PEG is widely used to produce Aqueous Two-Phase Systems (ATPS) in mixtures with salt, as potassium phosphate [16] and sodium succinate [17], or polymers, as dextrane [18,19], and to compartmentalize or precipitate biomolecules, as proteins [20-23] or nucleic acids [24]. The use of PEG as a reference osmolyte is a standard approach to study interactions and conformations of proteins [25-28] and viruses [29] in condensed solutions, since they readily phase separate into a protein (or virus) rich phase coexisting with a PEG rich solution.

When the partitioning of the two solutes is total, or nearly so, the osmotic pressure of PEG, which has been fully characterized [30,31], equals the osmotic pressure of the coexisting phase. Since PEG also phase separates with DNA, it has been used as a controlled osmolyte to measure the osmotic pressure of counterion condensed long DNA double helices [32], and across the isotropic-cholesteric and cholesteric-line hexatic phase boundary in solutions of long double stranded DNA [33,34].

Phase separation is also observed in mixed solutions of PEG and DNA oligomers [35-37] as well as in PEG and RNA oligomers [38] even for chains as short as 4 bases (unpublished data). This phenomenon has not been so far studied in detail. Phase separation of polymeric species is quite common, a phenomenon typically described in terms of the Flory-Huggins model, according to which the degree of polymerization is a key factor in the reduction of the entropy of mixing. When the degree of polymerization of one of the species is small, as in the case of PEG polymers and short DNA or RNA oligomers, the partitioning of the 
species might become partial.

When the phase separation between PEG and proteins or long DNA is "strong", with a nearly complete partitioning of the two molecular species between the two phases, the determination of the osmotic pressures is straightforward both because (i) the PEG concentration in the PEG-rich phase directly gauges the overall osmotic pressure and because (ii) the PEG concentration in the PEG-rich phase is simply determined from the initial concentrations and the volume fractions of the coexisting phases. When instead there is a partial partitioning between the species, both these steps must be reconsidered. For clarity, let's call phase 1 the PEG-rich phase and phase 2 the DNA-rich phase, and $\mathrm{c}_{\mathrm{DNA}, 1}, \mathrm{c}_{\mathrm{DNA}, 2}, \mathrm{c}_{\mathrm{PEG}, 1}$, and $\mathrm{c}_{\mathrm{PEG}, 2}$ the concentrations of DNA and PEG in phase 1 and phase 2, respectively (see Fig. 2). The presence of the minority components, $\mathrm{c}_{\mathrm{DNA}, 1}$ and $\mathrm{c}_{\mathrm{PEG}, 2}$ prevents from simply attributing all PEG to phase 1 and all DNA to phase 2 , as generally done when the osmotic pressure is measured via phase separation with PEG [32]. This issue will be discussed in Section 3, where we describe the experimental determination of all concentrations. At the same time, the presence of the minority components affects the osmotic pressure, as discussed in Section 4.

\section{PHASE DIAGRAMS IN MIXTURES OF PEG AND DNA OLIGOMERS}

We extensively investigated aqueous mixtures of DNA oligomers and PEG having overall concentrations of $\mathrm{c}_{\mathrm{DNA}}$ and $\mathrm{c}_{\mathrm{PEG}}$, respectively. We considered three different sequences of DNA and two PEG molecular weights, $8 \mathrm{kDa}$ and $20 \mathrm{kDa}$. DNA sequences have been chosen to provide duplex with end-to-end interactions of different kind and strength, as sketched in Fig. 2A. In particular, the "Dickerson Dodecamer", DD (5'-CGCGAATTCGCG-3'), is a self-complementary sequence that forms blunt-end duplexes that experience a mild end-to-end attraction because of the stacking interaction of their terminal paired bases. The DD duplex structure, of B-DNA type, has been well characterized by previous works [39]. We also considered modified version of DD, the "shifted Dickerson Dodecamer", sDD (5'-GCGAATTCGCGC-3'), obtained by relocating the first Cytosine nucleobase to the opposite (-3') end. SDD is thus selfcomplementary only in a subset of 10 bases, and thus forms duplexes with 2-base-long GC overhangs at the 3 '-end. Such unpaired bases (or "dangling ends") can pair with their counterpart of other sDD duplexes, thus acting as sticky-ends which drive the inter-duplex interaction. Both these sequences are composed of 12 nucleotides, therefore the length, $\mathrm{L}$, of the duplexes is $\mathrm{L} \approx 4.1 \mathrm{~nm}$. Given that the DNA helix diameter is approximately $\mathrm{D} \approx 2 \mathrm{~nm}$, the axial ratio of $\mathrm{DD}$ and $\mathrm{sDD}$ duplexes is $\mathrm{L} / \mathrm{D} \approx 2$. Both $\mathrm{DD}$ and $\mathrm{sDD} \mathrm{c}_{\mathrm{DNA}}-\mathrm{T}$ phase diagram has been already investigated, showing the formation of cholesteric $\left(\mathrm{N}^{*}\right)$, uniaxial columnar (COL) and crystalline columnar (C2) LC phases [1,2,7,40].

The third DNA sequence, DD-TT (5'-CGCGAATTCGCG-TT-3'), is a 14mer composed by the same DD sequence with the addition of two 3'-terminal Thymine nucleobases. The almost negligible T-T pairing affinity lowers inter-duplex interaction, in turn preventing the formation of $\mathrm{LC}$, while the axial ratio, $\mathrm{L} / \mathrm{D} \approx$ 2.4, is kept of the order of the other duplexes. In addition, we also tested the RNA analog of DD, pDD-RNA, which has the same sequence of DD except having Uraciles instead of Thymines, to test the universality of PEG-nucleic acid oligomers phase separation. Indeed, while DNA is most commonly a B-form helix, RNA duplexes have an A-form helical structure, in which the base pairs are tilted with respect to the helical axes. Because of this different helical geometry, the axial ratio of pDD-RNA duplexes is $\mathrm{L} / \mathrm{D} \approx 1.5$. Despite such structural differences and the presence of an additional phosphate at each 5'- terminus, similar LC behavior has been observed either for pDD-RNA alone [41] or mixed with PEG [38].

We have here investigated four different systems: DD + PEG 8kDa, 0.01M HEPES buffer $\mathrm{pH} 7.5$ and $300 \mathrm{mM} \mathrm{NaCl}$; pDD-RNA + PEG 8kDa, 0.01M HEPES buffer pH 7.5 and $60 \mathrm{mM} \mathrm{MgCl}_{2}$; sDD + PEG 20kDa with no buffer nor added salt; DD-TT + PEG 20kDa, with no buffer nor added salt. The different choices were related to the availability and storage condition of the samples. For each of these systems and for every choice of $\mathrm{c}_{\mathrm{DNA}}$ and $\mathrm{c}_{\mathrm{PEG}}$, samples were prepared in flat glass cells and observed in polarized optical microscopy (see materials and methods).

In all systems, at low enough PEG concentration, the solutions are homogeneous. Upon increasing PEG 
and DNA (or RNA) concentrations, phase separations are eventually observed in all systems, that split into a PEG-rich isotropic phase and a DNA-rich phase that can be either isotropic or liquid crystalline. We have thus classified the phase separations on the basis of the ordering of the DNA-rich phase: ISO-ISO, when DNA phase is isotropic, Fig. 2B; ISO-N*, when DNA phase shows $\mathrm{N}^{*}$ ordering, Fig. 2C; ISO-COL, when DNA phase shows COL ordering, Fig. 2D and ISO-C2, when DNA phase shows C2 ordering. Phase separation follows a mechanism of nucleation and growth process [35]. After the growth, taking place in a few minutes, and an equilibration period of 1-2 days, DNA-rich domains have sizes ranging from few to hundreds of microns. Their shape is typically circular at low PEG content, i.e. lower average viscosity, or branched at high PEG content.

Fig. 3 summarizes the observed phase behavior for the four systems, with the use of ternary phase diagrams. Each point on the ternary diagram uniquely indicates its DNA, PEG and water mass composition (w/w). This is illustrated in the construction in Fig. 3D for point "a", whose composition is 0.4 w/w DNA, $0.1 \mathrm{w} / \mathrm{w}$ PEG and $0.5 \mathrm{w} / \mathrm{w}$ water. All experimental observations are reported in the ternary diagrams, with black squares for isotropic mixtures, magenta pentagons for ISO-ISO phase separations, blue dots for ISO$\mathrm{N}^{*}$ phase separations, red triangles for ISO-COL phase separations and green diamonds for ISO-C2 phase separations. The empty symbols on the zero PEG axis are the experimental points describing the phase diagrams of DNA solutions with no PEG. Dotted lines, parallel to the water axes, indicate lines of constant DNA and dashed lines, parallel to the DNA axes, are lines of constant PEG. By comparing the four diagrams we can determine the general behavior of oligoDNA-PEG mixtures: (i) at very low DNA concentration, a large PEG w/w is required to induce phase separation (see line 1 in Fig. 3C); (ii) upon increasing the amount of DNA, the PEG w/w threshold for phase separation lowers, (see line 2 and 3 in Fig. 3C); (iii) there is a range of DNA concentrations at which all the phases are obtained by increasing PEG w/w. This is the case of the samples laying on line 4 in Fig. 3A $\left(\mathrm{c}_{\mathrm{DNA}}=200 \mathrm{~g} / \mathrm{l}\right)$ along which we have found ISO, $\mathrm{N}^{*}, \mathrm{COL}$ and $\mathrm{C} 2$ phases; (iv) at constant PEG w/w, phase separation is found above a threshold DNA w/w (see line 5 in Fig. 3C), which decreases as PEG w/w increases (see line 6 in Fig. 3C); (v) at high PEG w/w only highly ordered DNA phases survive, as $\mathrm{C} 2$ and amorphous crystals.

Having examined a large set of samples having different $\mathrm{c}_{\mathrm{DNA}}$ and $\mathrm{c}_{\mathrm{PEG}}$, we could approximately determine the binodal line as interpolation between the concentrations of samples that do and do not phase separate. Such interpolated concentrations are plotted in Fig. 4 as symbols. To provide an analytic expression to the binodal curves, we fitted the experimental data with the function $y=m_{1} \cdot \exp \left(m_{2} \cdot \sqrt{x}+m_{3} \cdot x^{3}\right)$ empirically proposed to describe the binodal curve of PEG - Potassium Phosphate solutions [20], where $\mathrm{y}=$ PEG $w / w$ and $x=$ DNA w/w. Since our data are gathered in the region of low DNA w/w, i.e. small $x$, the parameter $m_{3}$ has little relevance. We thus simplified the functional expression by setting $m_{3}=0$, obtaining

$$
c_{P E G}=m_{1} \cdot \exp \left(m_{2} \cdot \sqrt{c_{D N A}}\right)
$$

The binodal lines obtained in this way are shown in Fig. 4 as dashed lines and in the diagrams of Fig. 3 as black lines. By comparing the resulting binodal lines, it can be noticed that DD and pDD-RNA separate more easily than SDD and DD-TT since their binodal is shifted toward lower DNA and PEG content. This difference is probably due to the presence of added monovalent and divalent salts in the DD and pDD-RNA systems, respectively, in line with previous observations indicating that the formation of ATPS in PEG mixtures is promoted by higher ionic strength [20]. Moreover, the presence of cations has a strong effect on DNA: they generally reduce the lateral electrostatic repulsion between helices by screening the negative charges of the DNA backbone phosphates [42], while divalent and multivalent cations, as $\mathrm{Mg}^{2+}$ and spermine, favor DNA condensation [43-46].

\section{PARTITIONING OF DNA AND PEG}

The very existence of measurable binodal curves indicates that for the majority of the preparations the 
partitioning of PEG and DNA in phase 1 and 2 is not complete. By defining $\phi_{1}$ and $\phi_{2}$ as the fractions of the total volume occupied by phase 1 and 2, respectively, mass conservation of PEG and DNA implies that:

$$
\begin{aligned}
& c_{P E G}=c_{P E G, 1} \cdot \phi_{1}+c_{P E G, 2} \cdot \phi_{2} \\
& c_{D N A}=c_{D N A, 1} \cdot \phi_{1}+c_{D N A, 2} \cdot \phi_{2}
\end{aligned}
$$

where $\phi_{1}+\phi_{2}=1$. Since coexisting phases lay on the binodal curve, Eq. 1 holds for both phases, i.e. $c_{P E G, 1}=m_{1} \cdot \exp \left(m_{2} \cdot \sqrt{c_{D N A, 1}}\right)$ and $c_{P E G, 2}=m_{1} \cdot \exp \left(m_{2} \cdot \sqrt{c_{D N A, 2}}\right)$. This set of equations enables, at least in principle, determining the concentrations in both phases on the basis of $\mathrm{c}_{\mathrm{DNA}}$ and $\mathrm{c}_{\mathrm{PEG}}$ once $\phi_{1}$ and $\phi_{2}$ are known.

We experimentally measured $\phi_{1}$ and $\phi_{2}$ by adding a fraction of fluorescent PEG (FITC labelled PEG 5kDa) to the PEG stock solutions, at the 1:100 stoichiometric ratio between fluorescent PEG and the unlabelled PEG. Microscope images of the total sample area were collected and analyzed to extract the volume fractions of the two phases, as shown in Fig. 5A-C, (see materials and methods). In practice, the measured values of $\phi_{1}$ and $\phi_{2}$ do not enable to extract reliable values of the whole set of concentrations probably because the approximation on the "tails" of the binodal curve at large DNA content is too rude.

More direct information is obtained by analyzing the fluorescent emission of fluorinated PEG, as described in Fig. 5. The two peaks shape of the histogram of the fluorescence intensity, Fig. 5C, shows that while the fluorescence signal mostly comes from phase 1, rich in fluorescent PEG, there is still nonnegligible signal from phase 2 . The ratio of the average intensity measured in the two phases, $r=$ $\bar{I}_{P E G, 2} / \bar{I}_{P E G, 1}$, can be considered a direct measurement of $c_{P E G, 2} / c_{P E G, 1}$. Knowing $c_{P E G, 1}$ we calculate $c_{D N A, 1}$ from the expression of the binodal line, and then $c_{D N A, 2}$ is obtained from DNA mass conservation $c_{D N A, 2}=\left(c_{D N A, 0}-c_{D N A, 1} \cdot \phi_{1}\right) / \phi_{2}$.

By comparing the two approaches, we opted to use the direct measurement of $c_{P E G, 2} / c_{P E G, 1}$ for the samples with larger DNA content, while we used the approach based on the analytical expression of the binodal line at low DNA content, where the majority of the available experimental data lies, making the determination of the lines quite robust. Anyway, the two methods are highly compatible, confirming that analytical expression of the binodal line is a good description of the actual behavior of the system. Based on this analysis, we could draw the tie lines, as shown in Fig. 6 for a selection of the experimental points of the DD and sDD systems.

The main source of error is the measurement of the volume fractions $\phi$, which we estimated having the sensibility $\approx 0.5 \%$ of the total volume of the cell. Thus, the measurements of small $\phi$ are the ones most affected by the error. To avoid unrealistic behaviors, in the analysis of the samples with smallest $\phi$ we introduced the additional rule of setting the slopes of the tielines to be parallel to the closest one.

In this analysis it was necessary to convert $\mathrm{c}_{\mathrm{PEG}}$ and $\mathrm{c}_{\mathrm{DNA}}$ from mass density ( $\left.\mathrm{g} / \mathrm{l}\right)$ to weight ratios. Since we want here to compare our observation with models based on cylinders, we have not used the mass density of dry DNA, $\mathrm{d}_{\mathrm{DNA} \text {,dry }} \approx 1700 \mathrm{~g} / \mathrm{l}$ [47], but rather the mass density of hydrated DNA in a cylinder roughly enclosing the duplex, with a diameter of $1.95 \mathrm{~nm}$, which is much larger than the actual volume since it also includes the volume of the two grooves. This assumption leads to a mass density $\mathrm{d}_{\mathrm{DNA}} \approx 1100 \mathrm{~g} / \mathrm{l}$.

\section{OSMOTIC PRESSURE IN DNA LIQUID CRYSTALS}

Our results indicate that the phase separation in mixtures of PEG and DNA oligomers is a general phenomenon, which may also involve LC ordering of the DNA-rich phase. LC ordering is however not a necessary condition for phase separation, as demonstrated by liquid-liquid coexistence both in DD-TT solutions and in the DD solutions at low PEG. There are two basic mechanisms that could drive the phase separation. (i) The presence of attractive forces between DNA duplexes. Such interactions cannot but be between duplex terminal bases. Indeed, in the absence of polycations, side-side interaction between DNA 
double helices is always repulsive, and can be understood in terms of hydration and electrostatic forces [42]. Base stacking between the blunt ends of DD duplexes, and the mild attraction between the unpaired terminal bases of DD-TT, might in principle justify the phase separation. (ii) Entropic, depletion-type forces due to the difference in flexibility between the two species. The rigid DNA duplexes reduce the conformational space of PEG polymers when they are closer than their gyration radius. Thus, by phase separating, the system reduces the contacts between PEG and DNA, thus gaining phase space volume [50]. Which of the two mechanisms dominates the DNA-PEG phase separation is at the moment not clear.

At equilibrium, the osmotic pressure of the two phases must be the same, i.e. $\Pi_{1}=\Pi_{2}$. If the partitioning was complete, we could use $c_{\mathrm{PEG}, 1}$ to determine $\Pi^{\prime}=\Pi_{1}\left(\mathrm{c}_{\mathrm{PEG}, 1}\right)=\Pi_{2}\left(\mathrm{c}_{\mathrm{DNA}, 2}\right)$, where $\Pi_{1}\left(\mathrm{c}_{\mathrm{PEG}, 1}\right)$ has been obtained by the already known dependence of PEG osmotic pressure on its concentration [30,31]. П' values obtained in this way are shown in Fig. 7A as empty squares. However, the partitioning of PEG and DNA between the two phases is only partial. The concentrations of the minority components in the two phases, despite being smaller than those of the dominant components, could have a relevant effect on the osmotic pressures and their meaning.

Let's first consider the effect of the presence of DNA in phase 1. Since we can safely rule out attractive interactions between PEG and DNA molecules, we can consider DNA as dilute inclusions in a disordered polymer solution. As such, they reduce the free volume of PEG, which is thus effectively at a concentration larger than $\mathrm{C}_{\mathrm{PEG}, 1}$. The free volume subtracted from PEG includes the volume of the DNA duplexes surrounded by a depletion layer having a thickness of the order of the gyration radius of the PEG coils, which for the molecular weight here considered is $\mathrm{R}_{\mathrm{G}} \approx 3 \mathrm{~nm}(8 \mathrm{kDa})-5 \mathrm{~nm}(20 \mathrm{kDa})$ [51-53]. Since the size of each PEG coil in this study is comparable with the volume of a DNA duplex, we can adopt the simplifying notion that the decrease of the free volume of PEG equals the volume that the DNA duplexes would occupy if they were with no PEG but at the same osmotic pressure: $\Pi_{P E G, 1}\left(\frac{c_{P E G, 1}}{\phi_{P E G, 1}}\right) \approx \Pi_{C S}\left(f_{D N A, 1}\right)$, where we approximate the pressure of the DNA fraction with the CS. $f_{D N A, 1}$ is the DNA packing fraction which gives the equilibrium pressure. The volume available to the PEG fraction is $V_{P E G, 1} \approx V_{T O T}(1-$ $\left.\frac{\mathrm{c}_{D N A, 1}}{d_{D N A} f_{D N A, 1}}\right)$, so that $\phi_{P E G, 1}=V_{P E G, 1} / V_{T O T}$. In other words, we are here adopting the notion that both species in the mixture in phase 1 contribute to the osmotic pressure, in line with what observed and predicted for mixtures of hard spheres in the liquid state [54]. The resulting $\Pi^{\prime \prime}=\Pi_{P E G, 1}\left(\frac{c_{P E G, 1}}{\phi_{P E G, 1}}\right)$ is plotted in Fig. 7a as a function of $\mathrm{c}_{D N A, 2}$ as empty diamond. As visible, the presence of DNA in the PEG-rich phase enhances rather significantly the osmotic pressure at low pressures, when the partitioning is milder, while it becomes negligible at larger pressures, at which the partitioning is virtually complete.

Symmetrically, phase 2, rich in DNA, hosts a minority component of PEG, which also becomes less relevant at larger pressures. At the lowest pressures, phase 2 of DD is in the ISO phase, with $\mathrm{c}_{\mathrm{DNA}, 2} \approx 230$ $\mathrm{mg} / \mathrm{ml}$. This corresponds to a density of about one DNA duplexes every $60 \mathrm{~nm}^{3}$, in turn indicating a mean distance of about $4 \mathrm{~nm}$, clearly compatible with the presence of PEG polymers in the interstitial spaces. Upon increasing the osmotic pressure, the solutions develop cholesteric LC ordering with concentrations $\mathrm{c}_{\mathrm{DNA}, 2}$ in the range $260-420 \mathrm{mg} / \mathrm{ml}$. The mean intercolumnar distance, for this range of concentration, is 3-4 $\mathrm{nm}$, again fully compatible with the intercalation of PEG. At higher pressures, the system turns columnar, and the intercolumnar distance decreases further. This transition is accompanied by a reduction in $\mathrm{C}_{\mathrm{PEG}, 2}$ which becomes virtually negligible.

The effect on the osmotic pressure of PEG in phase 2 can be approximated by considering the fact that, at the interface between phase 1 and phase 2, PEG molecules within the interstitial spaces of DNA in phase 2 are in equilibrium with PEG molecules in phase 1. One could thus assume that volume per molecule of PEG in the interstitial spaces of DNA is equal to that in phase 1, i.e. that the local PEG concentration in both phases is equal to $\frac{c_{P E G, 1}}{\phi_{P E G, 1}}$, locally providing the same osmotic pressure. Therefore, the osmotic pressure of 
phase 2 includes the pressure from DNA, which is the quantity we wish to determine, plus the pressure of PEG, which is the same as the one in phase 1 but is exerted only where the interstitial spaces of DNA contact the interface. The surface fraction on which this occurs is given by the fraction of volume filled by PEG in phase 2, i.e. $\frac{\mathrm{c}_{P E G, 2} \phi_{P E G, 1}}{\mathrm{c}_{P E G, 1}}$. We can thus finally determine the osmotic pressure of DNA in phase 2 as the osmotic pressure on the interface between the coexisting phases, reduced by the pressure sustained by the PEG fraction in phase 2: $\Pi_{\mathrm{DNA}, 2}=\Pi^{\prime \prime}\left(1-\frac{\mathrm{c}_{P E G, 2} \phi_{P E G, 1}}{\mathrm{c}_{P E G, 1}}\right)$. These values are also plotted in Fig. 7A as filled dots. The values are in between the CS equation of state and the perfect gas line.

\section{DISCUSSION}

In Fig. 7B we show $\Pi_{\mathrm{DNA}, 2}$ for the different systems we have studied: DD (dots), sDD (triangles) and DD-TT (squares). Black symbols indicate liquid-liquid phase separations, i.e. phase coexistence in which the DNA-rich phase is in the ISO phase. These are available only for DD at the lowest concentration at which we could find phase separation, and for DD-TT, for which just two experimental points are available (the second located at $\beta \Pi D^{3} \approx 3.9$ and $\mathrm{c}_{\mathrm{DNA}} \approx 570 \mathrm{mg} / \mathrm{ml}$ is not shown because it's out the graph limits) since at lower concentration it does not phase separate. These two points lay closest to the CS equation of state, as expected, since in these cases attractive interactions do not play an important role.

As the LC phases appear, the osmotic pressure vs. concentrations curve move further away from the CS equation of state, as expected because of the larger role played by the attractive interactions and by the increase of free volume due to better packing. In Fig. 7B we also plot the predictions of the computer simulation of Ref. [11], already shown in Fig. 1. Here the predictions have been expressed in terms of DNA concentration by using the density $\mathrm{d}_{\mathrm{DNA}}$ as discussed above. The data are approximated rather nicely by the coarse-grained model, a condition that enables us to use the simulation to give an estimate of the end-to-end binding energy. As visible, the data behaves as the simulations performed with potential depth $\mathrm{E}$ in between 10 and $12 \mathrm{k}_{\mathrm{B}} \mathrm{T}$, which would correspond to a binding free energy of $\Delta \mathrm{G} \approx 6 \mathrm{kcal} / \mathrm{mol}$. This number is a bit large with respect to expectations but not unreasonable.

In comparing sDD and DD data we need also to consider the different ionic strength and $\mathrm{pH}$ of these two systems, which are both known to influence the phase separation. Indeed, while sDD has no added salt, DD solutions contain $300 \mathrm{mM} \mathrm{NaCl}$ and additional $\mathrm{Na}^{+}$ions from HEPES buffer. Since salt addition promotes ATPS formation with PEG [20,21] its concentration in phase 2 could in principle be even higher. Added $\mathrm{NaCl}$ is known to screen the negative charges of the DNA phosphate backbone and to lower the osmotic pressure of long DNA at low $\mathrm{c}_{\mathrm{DNA}}$, a regime where the screened electrostatic is the dominating interaction [42]. For this reason the extracted pressure for DD could be under-estimated in our present work, this leading to the over-estimation of the stacking $\Delta \mathrm{G}$.

In discussing this result for $\Delta \mathrm{G}$, it should first be mentioned that the simulation was performed with cylindrical duplexes and with an inter-duplex potential having a simple dependence on distance and angles, both of which are necessarily oversimplifications of the real systems. Thus, mapping the energy used for the simulations (which correspond to the interaction potential at contact) directly into the DNA duplex end-toend interaction free energy might also be an oversimplification.

The interactions between sDD duplexes, due to the pairing of the sticky overhangs, can be estimated by using the nearest neighbor model for DNA hybridization, in analogy to what has been done in Ref. [3] for the GCCG system, which comes out to be about $4 \mathrm{kcal} / \mathrm{mol}$. The interaction between DD is instead due to stacking forces only. Clean measurements of inter-duplex stacking are still missing. Direct measurements were performed for the interaction between bundles of helices in complex nanostructures [9], obtaining $\Delta \mathrm{G} \approx$ $3.4 \mathrm{kcal} / \mathrm{mol}$. In this case, however, duplex don't have the freedom of adjusting their mutual azimuthal angle. Indirect determinations have been performed by the modelling of LC phase diagrams [7] obtaining $\Delta \mathrm{G} \approx 3.5$ $\mathrm{kcal} / \mathrm{mol}$. In this case, though, the evaluation heavily depends on the use of another coarse-grained model 
[12]. The stacking interaction between blunt-ended duplexes has been evaluated by atomistic computer simulation by Maffeo et al. [10]. Their result is $\Delta \mathrm{G} \approx 6 \mathrm{kcal} / \mathrm{mol}$, but it is generally believed that stacking is overestimated by this computational approach [55]. They also find that the variation in free energy when the azimuthal angle is changed is quite consistent, of the order of $2 \mathrm{kcal} / \mathrm{mol}$, indicating that the free adjustment of this parameter, enabled by the free axial rotation of duplexes in the LC phases, might have a large effect on the inter-duplex interactions.

\section{CONCLUSIONS}

We investigated the formation of aqueous two-phase systems in mixtures of PEG and DNA (RNA) duplexforming oligomers. We found the conditions in which the homogeneous solutions phase separate into coexisting PEG-rich and DNA-rich phases, the latter hosting isotropic, $\mathrm{N}^{*}, \mathrm{COL}$ or $\mathrm{C} 2$ phases. We determined the PEG/DNA phase diagrams for duplexes having different end-to-end interactions - blunt ends (DD and pDD-RNA), sticky overhangs (sDD), aggregation-preventing overhangs (DD-TT) - and different ionic strengths. The resulting binodal lines are well described by the analytical expression empirically obtained for PEG-phosphate ATPS [20].

By measuring the partitioning of the molecular species in the two coexisting phases, we determined the osmotic pressure of DNA duplexes, $\Pi_{\mathrm{DNA}, 2}$, as a function of the $\mathrm{c}_{\mathrm{DNA}}$ across the phase diagram, for both isotropic and LC phases. We compared the equation of state obtained in this way with both the CarnahanStarling equation of state for hard spheres and with the pressure predicted by computer simulations of a system of aggregating cylinders [11]. We obtained a good agreement between experiments and simulations, and end-to-end attraction energies of the order of $6 \mathrm{kcal} / \mathrm{mol}$, a bit larger than expected, but still in agreement with the current models for DNA-DNA interactions [10,12].

The present work describes a simple and quick method for measuring the osmotic pressure, $\Pi$, and the concentration, $\mathrm{c}_{\mathrm{DNA}}$, of DNA LC phases, setting the basis for the usage of DNA/PEG ATPS to finely control the pressures and concentrations of the LC domains. DNA/PEG ATPS could provide an effective strategy to smoothly adjust properties of DNA liquid crystals which are currently hard to control, such as the cholesteric pitch of $\mathrm{N}^{*}$, in turn allowing more accurate measurement of DNA elastic coefficients and non-linear optical response [56,57].

\section{Materials and methods}

Materials. Lyophilized DNA and RNA oligonucleotides, HPLC purified, are from IDT (DD and pDD-RNA) and from Primm s.r.l. (sDD and DD-TT), while PEG 8kDa and 20kDa are from Sigma. Fluorescent PEG $5 \mathrm{kDa}$ is from Nanocs. Stock $50 \mathrm{mg} / \mathrm{ml}$ DNA/RNA solutions were obtained by weighting the desired DNA/RNA mass (few mg), directly in a $1.5 \mathrm{~mL}$ plastic Eppendorf tube, with a Kern ABT 100-5M scale, $0.01 \mathrm{mg}$ sensitivity, and adding the required milliQ water volume. Stock PEG solutions, from 0.05 to 0.3 $\mathrm{w} / \mathrm{w}$, were prepared by weighting the desired PEG mass ( 0.5 to $3 \mathrm{~g})$ and adding the required milliQ water volume in plastic falcon tubes to achieve a $10 \mathrm{~g}$ total solution weight.

Samples preparation. Mixtures of DNA and PEG were prepared obtained by depositing controlled volumes ( 0.5 to $1 \mu \mathrm{l})$ of stock DNA aqueous solution on a microscope glass slide, and letting them evaporate to obtain the desired DNA mass, $\mathrm{w}_{\mathrm{DNA}}$. Controlled volumes of PEG solution with mass fraction from 0.05 to $0.30 \mathrm{w} / \mathrm{w}$ and with the desired ionic strength and buffers, were added then and mixed with a needle. The resulting mixtures were rapidly covered with a second glass slide, sticked together with epoxy glue and sealed with fluorinated oil to ensure stability over time. Cell thickness is set to $20 \mu \mathrm{m}$ by the use of silica spacers.

Microscope images and analysis. Samples observation and analysis were performed with a Nikon TE-300 microscope allowing bright field, polarized and fluorescence configurations. Images were taken with a 
Nikon DS-Fi3 color CCD camera (2880 x 2048 pixels). Single low magnification images (2X objective), and when necessary multiple grid-stitched higher magnification images, were analyzed with Fiji-ImageJ software. To obtain the volume fractions of the phases, the area fraction of the fluorescent region and of the total sample area of 8-bit greyscale converted images were measured, after background subtraction, brightness/contrast adjustment and setting of the appropriate threshold.

Authors Contributions: T.P.F. and T.B. conceived the experiments; S.D.L., M.T. and T.P.F. performed the experiments; T.P.F. and T.B. analyzed the data; T.P.F., M.T. and T.B. wrote the manuscript.

Acknowledgments: We wish to thank M. Glaser for sharing the simulations data, and G.P. Smith, G. Zanchetta and N.A. Clark for useful discussions. M.T. acknowledges the support of the Invernizzi Fundation.

The authors declare that they have no competing interests. 


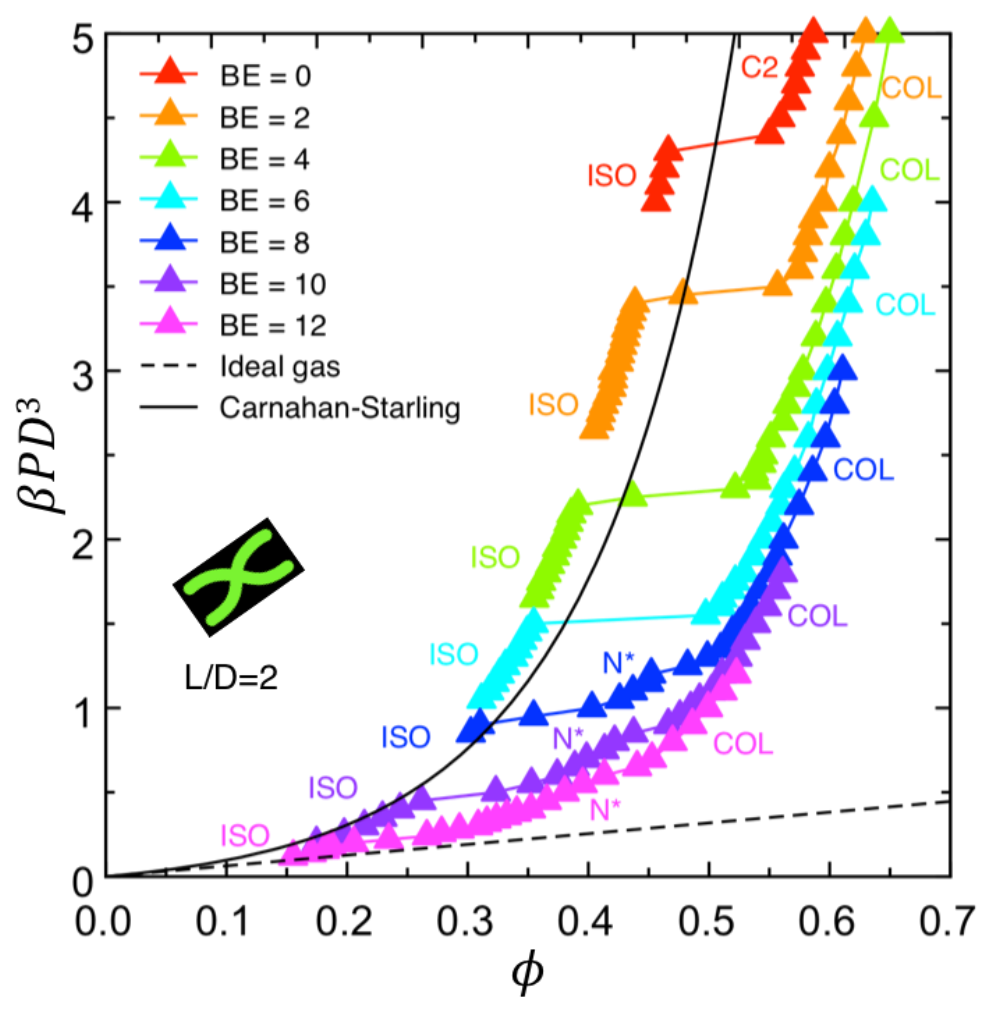

Fig. 1: Equation of state for a system of sticky cylinders of diameter $D$ and axial ratio $L / D=2$, expressed in dimensionless pressure, $\beta \mathrm{PD}^{3}$, where $\beta^{-1}=\mathrm{k}_{\mathrm{B}} \mathrm{T}$, vs. volume fraction $\phi$. The end-to-end binding energy $\mathrm{E}$ ranges from 0 to $12 \mathrm{k}_{\mathrm{B}} \mathrm{T}$. Data obtained from Monte Carlo simulations, adapted with permission from [11]. Colored triangles correspond to different energies, see legend, and phases are as indicated by colored labels, ISO $=$ isotropic, $\mathrm{N}^{*}=$ cholesteric, $\mathrm{COL}=$ columnar and $\mathrm{C} 2=$ columnar crystal. Lines connecting the symbols are drawn to help the identification of the first-order phase transitions, which take place at constant pressure. Solid black line is the Carnahan-Starling equation of state for hard spheres and dashed black line is the ideal gas equation. 


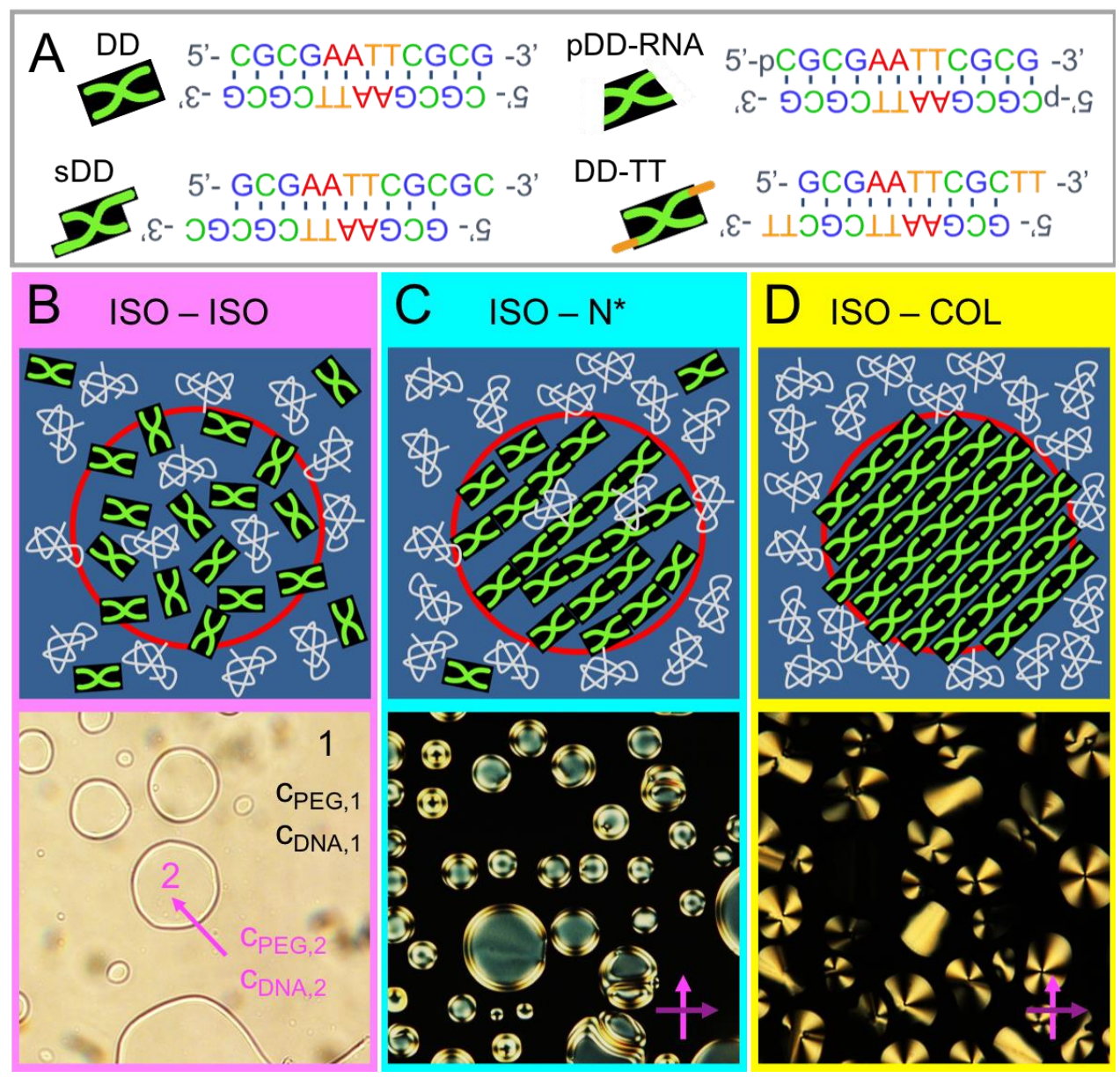

Fig. 2: (A) Sketches and sequences of the DNA and RNA duplexes considered in the present work: bluntend DD and RNA 5'phosphate DD, sticky-end SDD and non-sticky-end DD-TT, with corresponding sequences. (B-D upper panels) Pictorial representation of ATPS in mixtures of duplexes and PEG, sketched as grey bundles. Depending on concentrations, the system separates into a PEG-rich phase (phase 1) and a DNA-rich phase (phase 2), where $\mathrm{c}_{\mathrm{PEG}, 1}, \mathrm{c}_{\mathrm{DNA}, 1}$ and $\mathrm{c}_{\mathrm{PEG}, 2}, \mathrm{c}_{\mathrm{DNA}, 2}$ are the concentrations of PEG and DNA in phase 1 and phase 2, respectively. Upon increasing PEG and DNA concentrations, the partitioning of the two species becomes more effective, with progressively decreasing amount of DNA in phase 1 and PEG in phase 2. (B-D lower panels) Bright field micrograph of ISO-ISO phase separation (B), polarized optical micrographs of ISO-N* (C) and ISO-COL (D) phase separations. 


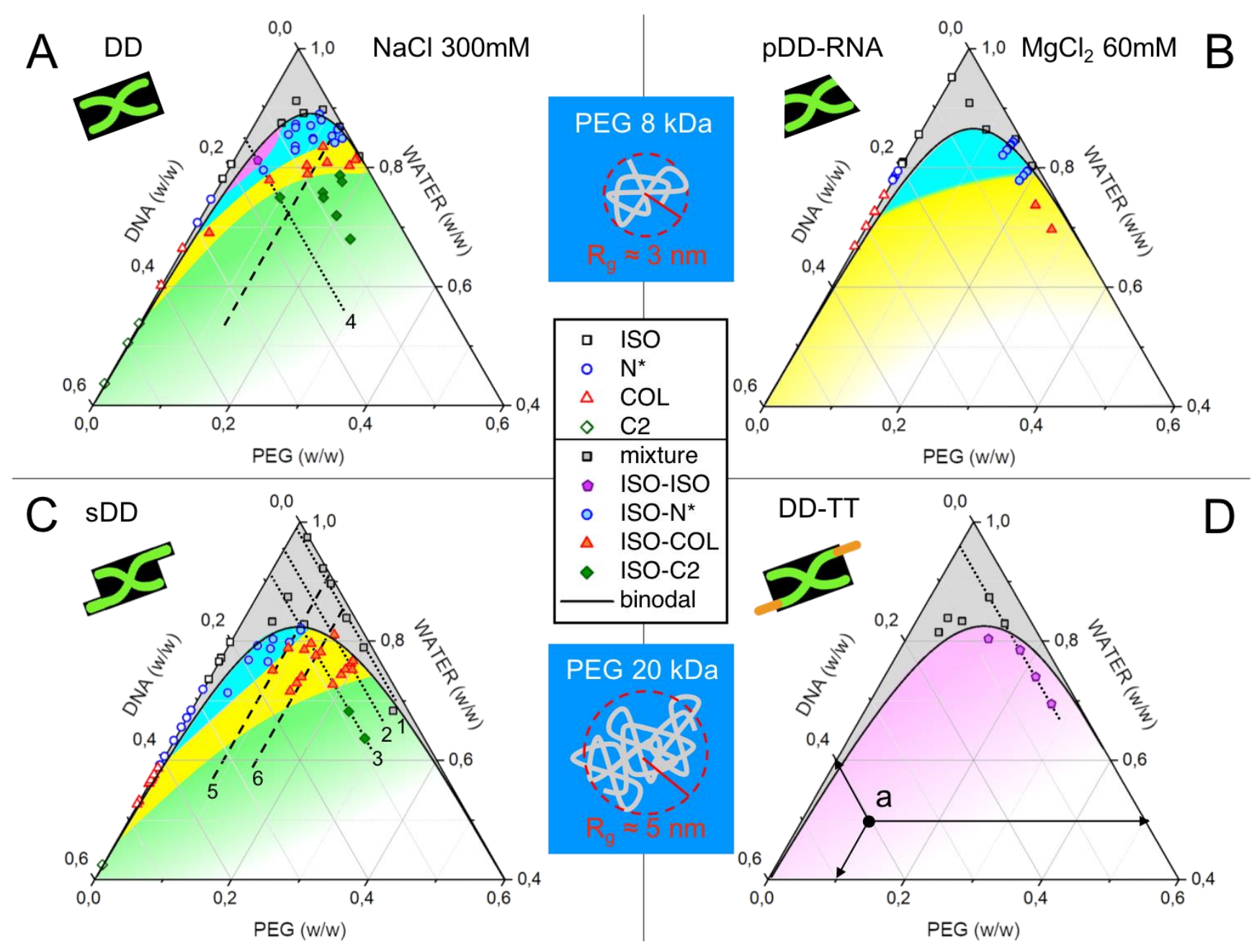

Fig. 3: Ternary diagrams of the four investigated mixtures in unities of mass fraction $w / w$ (i.e. $\mathrm{w}_{\mathrm{DNA}} / \mathrm{w}_{\mathrm{TOT}}$, $\mathrm{w}_{\mathrm{PEG}} / \mathrm{w}_{\mathrm{TOT}}$ and $\mathrm{w}_{\mathrm{WATER}} / \mathrm{w}_{\mathrm{TOT}}$, where $\mathrm{w}_{\mathrm{TOT}}=\mathrm{w}_{\mathrm{DNA}}+\mathrm{w}_{\mathrm{PEG}}+\mathrm{w}_{\mathrm{WATER}}$. (A) DD/PEG-8kDa/NaCl-300mM, (B) pDD-RNA /PEG-8kDa/MgCl 2 -60mM, (C) sDD/PEG-20kDa/water and (D) DD-TT/PEG-20kDa/water. Point "a" in panel (D) and arrows provide an example for the reading of the system composition (0.4 DNA w/w $0.1 \mathrm{PEG}$ w/w -0.5 water w/w). Symbols within the diagram indicate the initial samples preparations and their colors indicate the type of separation observed, according to the legend. Symbols laying on the axes of 0 PEG w/w indicate the phase diagrams of the pure DNA/RNA solution at room temperature. Solid black lines are the binodal lines, determined as described in the text, separating the region of homogeneous solution (grey shading) and the phase-coexistence region (violet, blue, yellow and green shading for ISOISO, ISO-N*, ISO-COL and ISO-C2 coexistence, respectively). Dotted lines indicate constant DNA preparations and dashed lines indicate constant PEG preparations. 


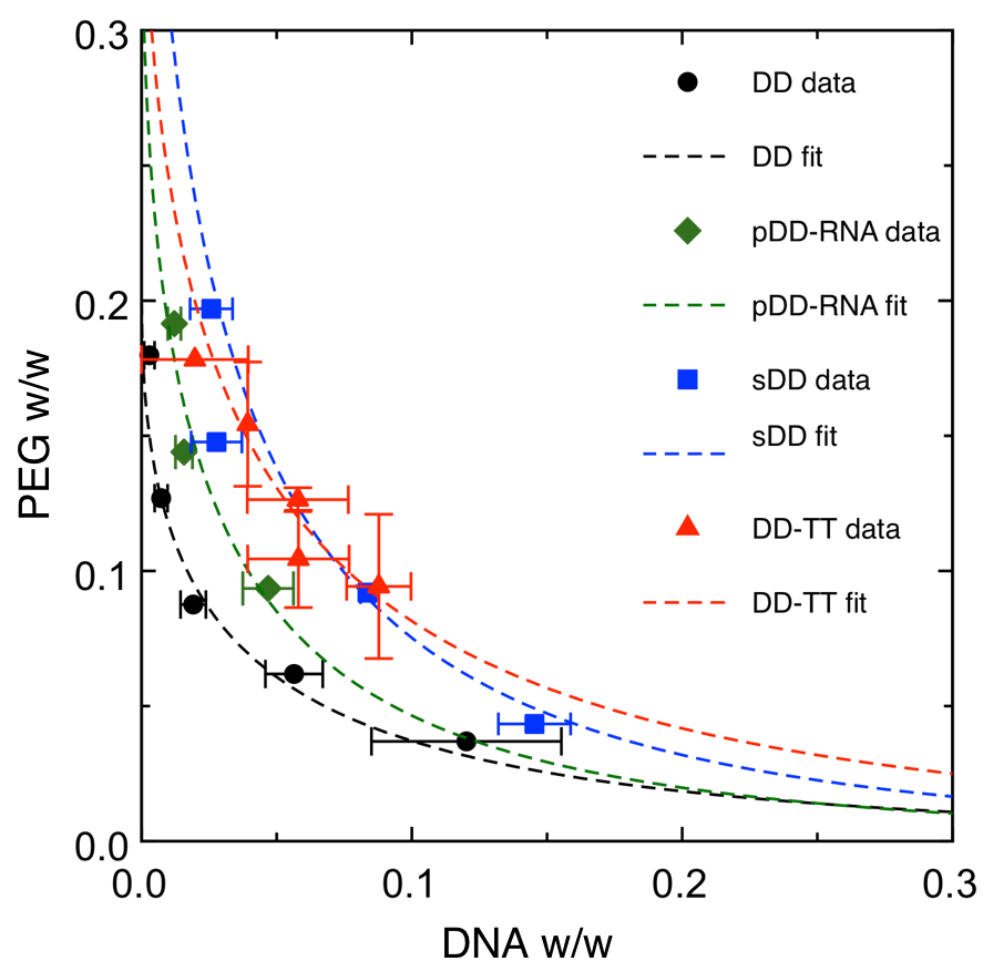

Fig. 4: Experimental determination of the binodal lines in PEG w/w vs DNA w/w diagram. Points are obtained as interpolation of the compositions of pairs of samples laying on the opposite sides of the binodal line. Error bars indicate the distance in $\mathrm{c}_{\mathrm{PEG}}$ and $\mathrm{c}_{\mathrm{DNA}}$ between the pairs. Dashed lines are the binodal obtained by fitting the points with the function $c_{P E G}=m_{1} \cdot \exp \left(m_{2} \cdot \sqrt{c_{D N A}}+m_{3} \cdot c_{D N A}{ }^{3}\right)$ from Ref. [20], simplified by assuming $\mathrm{m}_{3}=0$. Binodals closest to the axes indicate stronger partitioning of PEG and DNA between the phases. 


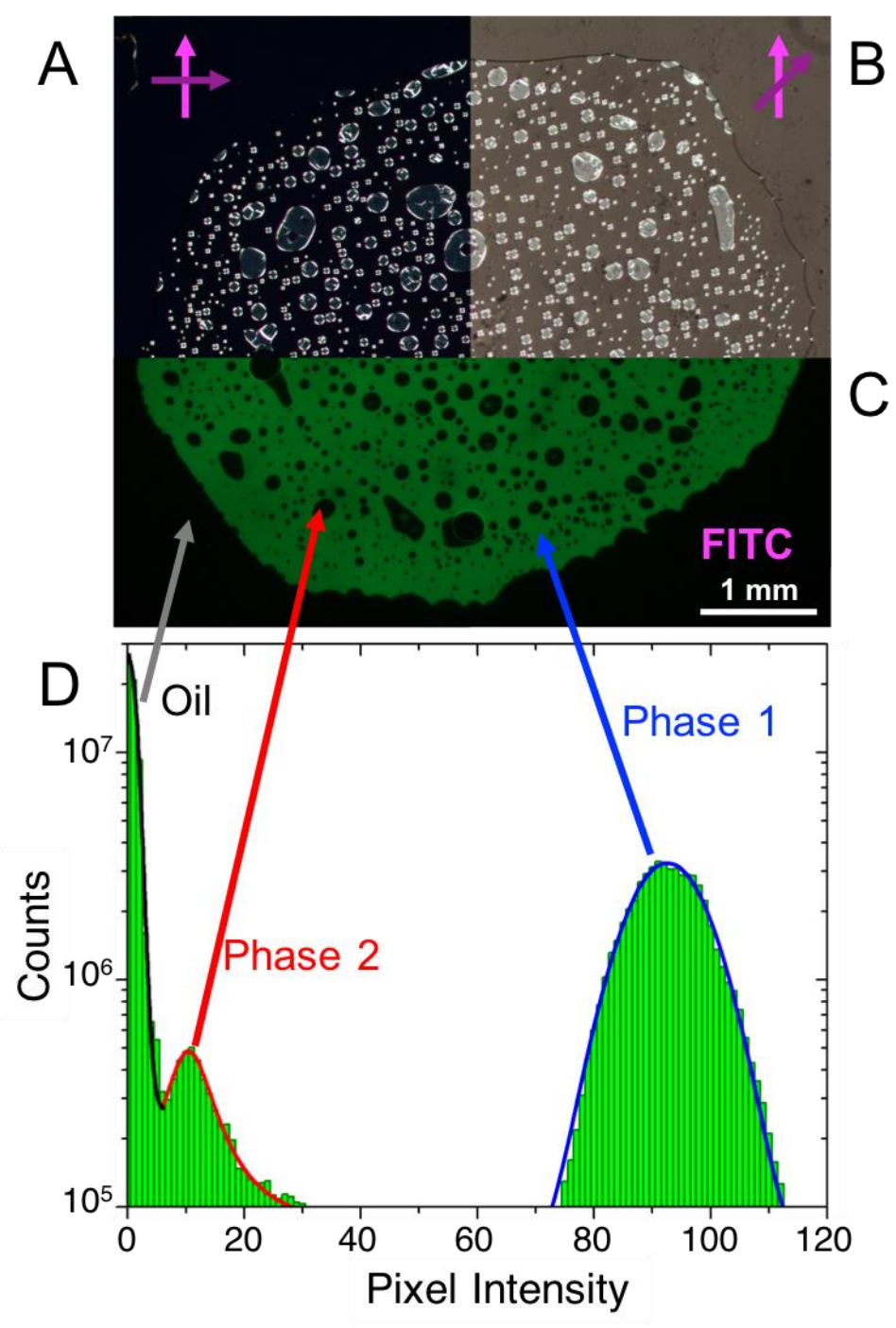

Fig. 5: Low magnification $(2 x)$ optical microscopy images of ISO-N* phase separation in DD/PEG$8 \mathrm{kDa} / \mathrm{NaCl}-300 \mathrm{mM}$ with $\mathrm{PEG} \mathrm{w} / \mathrm{w}=0.082$ and DNA $\mathrm{w} / \mathrm{w}=0.089$, with crossed polarizers $(\mathrm{A})$, partially uncrossed polarizers (B) and fluorescent emission (C). Fluorescent PEG 5kDa, tagged with FITC fluorophore, has been mixed to untagged PEG at 1:100 stoichiometric ratio. Fluorescence signal enables direct measurement of the volume fractions. In panel $\mathrm{C}, \phi_{1}=0.80 \pm 0.05$ and $\phi_{2}=0.20 \pm 0.05$. (D) Histogram of the fluorescence intensity measured on the 8-bit gray-scale image (black $=0$, white $=255$ ). The three peaks are associated to the fluorescent emission of: the oil background area (black contour peak and grey arrow), the PEG rich phase 1 (blue contour and blue arrow) and the DNA rich phase 2 (red contour and red arrow). The ratio of the mean intensity of phase 1 and phase 2, after subtraction of the oil background, gives the $\mathrm{c}_{\mathrm{PEG}, 1} / \mathrm{c}_{\mathrm{PEG}, 2}$ ratio, a value necessary to determine the composition of the two phases (see the text). 

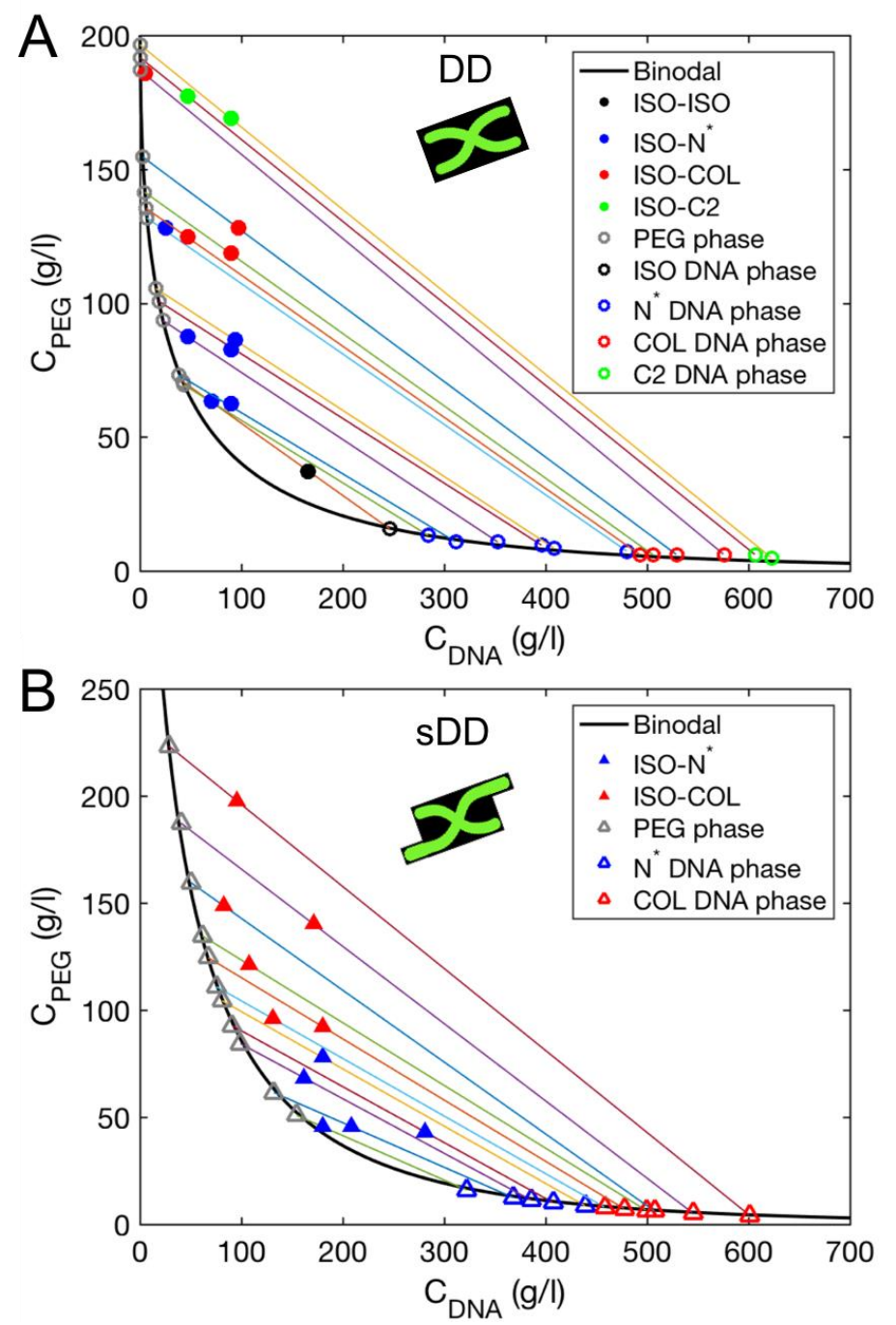

Fig. 6: Phase diagrams and phase compositions of DD/PEG-8kDa/NaCl-300mM (A) and sDD/PEG$20 \mathrm{kDa} / \mathrm{MgCl}_{2}-60 \mathrm{mM}$ (B). Full symbols are the initial preparations of a selected subsets of samples. Open symbols express the partitioning of the two components in the two phases: isotropic, PEG-rich phase 1 (grey symbols) and DNA-rich phase 2, which can be in ISO (black symbols), $\mathrm{N}^{*}$ (blue symbols), COL (red symbols) and C2 (green symbols) phases. Black lines are binodal lines. Colored lines are tie lines, connecting the initial preparation to the compositions of the coexisting phases. 

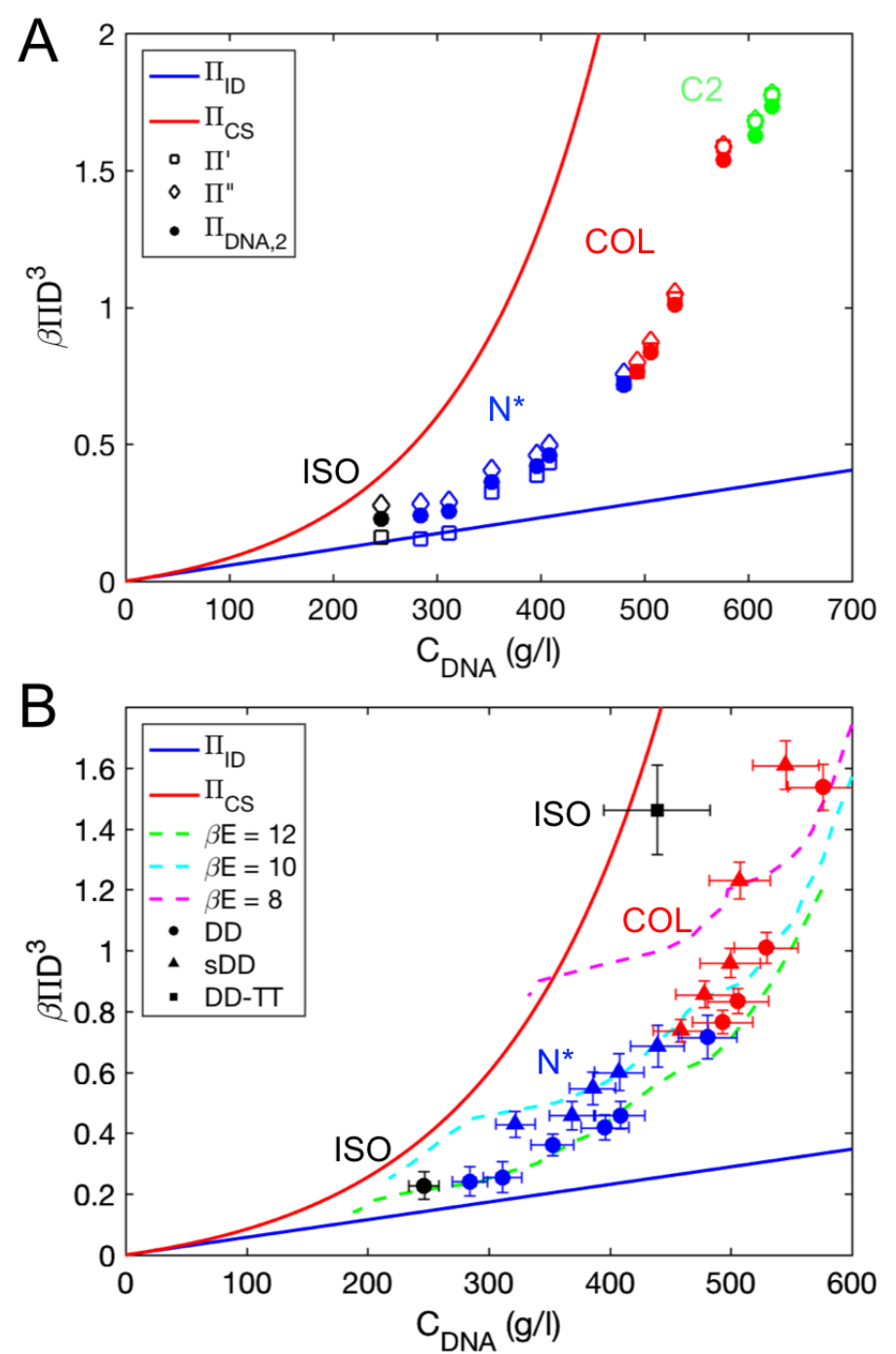

Fig. 7: (A) Dimensionless osmotic pressure, $\beta \Pi D^{3}$, as a function of DNA concentration, $c_{\mathrm{DNA}}(\mathrm{g} / \mathrm{l})$, measured for DD. $\Pi$ ' (empty squares), $\Pi$ " (open diamonds) and $\Pi_{\mathrm{DNA}, 2}$ (filled circles) indicate the three steps we have adopted for our final estimate of the osmotic pressure in phase 2. $\Pi$ ' is the osmotic pressure of PEG in phase 1. $\Pi$ " includes the free volume correction. $\Pi_{\mathrm{DNA}, 2}$ takes also into account the contribution of the residual PEG in phase 2. (B) Comparison of the experimental equation of state obtained for DD (dots), sDD (triangles) and DD-TT (squares). Error bars are obtained by propagating the experimental uncertainty on the measurement of $\phi$. Dashed lines are the prediction of the simulation for a system of sticky cylinders with binding energies as indicated in the legend, from Ref. [11]. (A, B) LC phases are marked as it follows: ISO = isotropic (black), $\mathrm{N}^{*}=$ cholesteric (blue), $\mathrm{COL}=$ columnar (red) and $\mathrm{C} 2=$ crystalline columnar (green). Solid red line is the Carnahan-Starling equation of state for hard spheres and blue line is the ideal gas equation. 
1. Nakata, M.; Zanchetta, G.; Chapman, B. D.; Jones, C. D.; Cross, J. O.; Pindak, R.; Bellini, T.; Clark, N. A. End-to-end stacking and liquid crystal condensation of 6 to 20 base pair DNA duplexes. Science 2007, 318, 1276-1279, doi:10.1126/science.1143826.

2. Zanchetta, G.; Nakata, M.; Buscaglia, M.; Clark, N. A.; Bellini, T. Liquid crystal ordering of DNA and RNA oligomers with partially overlapping sequences. J. Phys. Condens. Matter 2008, 20, 494214, doi:10.1088/0953-8984/20/49/494214.

3. Fraccia, T. P.; Smith, G. P.; Bethge, L.; Zanchetta, G.; Nava, G.; Klussmann, S.; Clark, N. A.; Bellini, T. Liquid Crystal Ordering and Isotropic Gelation in Solutions of Four-Base-Long DNA Oligomers. ACS Nano 2016, 10, 8508-8516, doi:10.1021/acsnano.6b03622.

4. Fraccia, T. P.; Smith, G. P.; Clark, N. A.; Bellini, T. Liquid crystal ordering of four-base-long DNA oligomers with both G-C and A-T pairing. Crystals 2018, 8, doi:10.3390/cryst8010005.

5. Salamonczyk, M.; Zhang, J.; Portale, G.; Zhu, C.; Kentzinger, E.; Gleeson, J. T.; Jakli, A.; De Michele, C.; Dhont, J. K. G.; Sprunt, S.; Stiakakis, E. Smectic phase in suspensions of gapped DNA duplexes. Nat. Commun. 2016, 7, 1-9, doi:10.1038/ncomms 13358.

6. Bellini, T.; Zanchetta, G.; Fraccia, T. P.; Cerbino, R.; Tsai, E.; Smith, G. P. Liquid crystal selfassembly of random- sequence DNA oligomers. Proc. Natl. Acad. Sci. U. S. A. 2012, 109, 11101115, doi:10.1073/pnas.1117463109.

7. Smith, G. P.; Fraccia, T. P.; Todisco, M.; Zanchetta, G.; Zhu, C.; Hayden, E.; Bellini, T.; Clark, N. A. Backbone-free duplex-stacked monomer nucleic acids exhibiting Watson - Crick selectivity. Proc. Natl. Acad. Sci. U. S. A. 2018, doi:10.1073/pnas.1721369115.

8. Yakovchuk, P.; Protozanova, E.; Frank-Kamenetskii, M. D. Base-stacking and base-pairing contributions into thermal stability of the DNA double helix. Nucleic Acids Res. 2006, 34, 564-574, doi:10.1093/nar/gkj454.

9. Kilchherr, F.; Wachauf, C.; Pelz, B.; Rief, M.; Zacharias, M.; Dietz, H. Single-molecule dissection of stacking forces in DNA. Science (80-. ). 2016, 353.

10. Maffeo, C.; Luan, B.; Aksimentiev, A. End-to-end attraction of duplex DNA. Nucleic Acids Res. 2012, 40, 3812-3821.

11. Kuriabova, T.; Betterton, M. D.; Glaser, M. a. Linear aggregation and liquid-crystalline order: comparison of Monte Carlo simulation and analytic theory. J. Mater. Chem. 2010, 20, 10366, doi:10.1039/c0jm02355h.

12. De Michele, C.; Rovigatti, L.; Bellini, T.; Sciortino, F. Self-assembly of short DNA duplexes: from a coarse-grained model to experiments through a theoretical link. Soft Matter 2012, 8, 8388-8398, doi:10.1039/c2sm25845e.

13. De Michele, C.; Bellini, T.; Sciortino, F. Self-assembly of bifunctional patchy particles with anisotropic shape into polymers chains: Theory, simulations, and experiments. Macromolecules 2012 , 45, 1090-1106.

14. Saurabh, S.; Lansac, Y.; Jang, Y. H.; Glaser, M. A.; Clark, N. A.; Maiti, P. K. Understanding the origin of liquid crystal ordering of ultrashort double-stranded DNA. Phys. Rev. E 2017, 95, 1-6, doi:10.1103/PhysRevE.95.032702.

15. Carnahan, N. F.; Starling, K. E. Equation of state for nonattracting rigid spheres. J. Chem. Phys. 1969, 51, 635-636, doi:10.1063/1.1672048.

16. Kaul, A.; Pereira, R. A. M.; Asenjo, J. A.; Merchuk, J. C. Kinetics of phase separation for polyethylene glycol-phosphate two-phase systems. Biotechnol. Bioeng. 1995, 48, 246-256, doi:10.1002/bit.260480311.

17. Raja, S.; Murty, V. R. Liquid-Liquid Equilibrium of Poly ( Ethylene Glycol ) $6000+$ Sodium Succinate + Water System at Different Temperatures. 2013, 2013.

18. Grossmann, C.; Tintinger, R.; Zhu, J.; Maurer, G. Aqueous two-phase systems of poly(ethylene glycol) and dextran - experimental results and modeling of thermodynamic properties. Fluid Phase Equilib. 1995, 106, 111-138, doi:10.1016/0378-3812(94)02626-C.

19. Zhao, Z.; Li, Q.; Ji, X.; Dimova, R.; Lipowsky, R.; Liu, Y. Molar mass fractionation in aqueous twophase polymer solutions of dextran and poly(ethylene glycol). J. Chromatogr. A 2016, 1452, 107115, doi:10.1016/j.chroma.2016.04.075.

20. Merchuk, J. C.; Andrews, B. A.; Asenjo, J. A. Aqueous two-phase systems for protein separation: Studies on phase inversion. J. Chromatogr. B Biomed. Sci. Appl. 1998, 711, 285-293, doi:10.1016/S0378-4347(97)00594-X.

21. Asenjo, J. A.; Andrews, B. A. Aqueous two-phase systems for protein separation: A perspective. $J$. 
Chromatogr. A 2011, 1218, 8826-8835, doi:10.1016/j.chroma.2011.06.051.

22. Ferreira, A. M.; Faustino, V. F. M.; Mondal, D.; Coutinho, J. A. P.; Freire, M. G. Improving the extraction and purification of immunoglobulin $\mathrm{G}$ by the use of ionic liquids as adjuvants in aqueous biphasic systems. J. Biotechnol. 2016, 236, 166-175, doi:10.1016/j.jbiotec.2016.08.015.

23. Monterroso, B.; Zorrilla, S.; Sobrinos-Sanguino, M.; Keating, C. D.; Rivas, G. Microenvironments created by liquid-liquid phase transition control the dynamic distribution of bacterial division FtsZ protein. Sci. Rep. 2016, 6, 1-13, doi:10.1038/srep35140.

24. Poudyal, R. R.; Pir Cakmak, F.; Keating, C. D.; Bevilacqua, P. C. Physical Principles and Extant Biology Reveal Roles for RNA-Containing Membraneless Compartments in Origins of Life Chemistry. Biochemistry 2018, 57, 2509-2519, doi:10.1021/acs.biochem.8b00081.

25. Parsegian, V. A.; Rand, R. P.; Rau, D. C. [3] Macromolecules and water: Probing with osmotic stress. Methods Enzymol. 1995, 259, 43-94, doi:10.1016/0076-6879(95)59039-0.

26. Annunziata, O.; Asherie, N.; Lomakin, A.; Pande, J.; Ogun, O.; Benedek, G. B. Effect of polyethylene glycol on the liquid-liquid phase transition in aqueous protein solutions. Proc. Natl. Acad. Sci. U. S. A. 2002, 99, 14165-14170, doi:10.1073/pnas.212507199.

27. Soranno, A.; Koenig, I.; Borgia, M. B.; Hofmann, H.; Zosel, F.; Nettels, D.; Schuler, B. Singlemolecule spectroscopy reveals polymer effects of disordered proteins in crowded environments. Proc. Natl. Acad. Sci. 2014, 111, 4874-4879, doi:10.1073/pnas.1322611111.

28. Pasquier, C.; Beaufils, S.; Bouchoux, A.; Rigault, S.; Cabane, B.; Lund, M.; Lechevalier, V.; Le Floch-Fouéré, C.; Pasco, M.; Pabœuf, G.; Pérez, J.; Pezennec, S. Osmotic pressures of lysozyme solutions from gas-like to crystal states. Phys. Chem. Chem. Phys. 2016, 18, 28458-28465, doi:10.1039/c6cp03867k.

29. Nurmemmedov, E.; Castelnovo, M.; Catalano, C. E.; Evilevitch, A. Biophysics of viral infectivity: Matching genome length with capsid size. Q. Rev. Biophys. 2007, 40, 327-356, doi:10.1017/S0033583508004666.

30. Stanley, C. B.; Strey, H. H. Measuring osmotic pressure of poly(ethylene glycol) solutions by sedimentation equilibrium ultracentrifugation. Macromolecules 2003, 36, 6888-6893, doi:10.1021/ma034079e.

31. Cohen, J. A.; Podgornik, R.; Hansen, P. L.; Parsegian, V. A. A phenomenological one-parameter equation of state for osmotic pressures of PEG and other neutral flexible polymers in good solvents. J. Phys. Chem. B 2009, 113, 3709-3714, doi:10.1021/jp806893a.

32. Rau, D. C.; Parsegian, V. A. Direct measurement of the intermolecular forces between counterioncondensed DNA double helices. Evidence for long range attractive hydration forces. Biophys. $J$. 1992, 61, 246-259, doi:10.1016/S0006-3495(92)81831-3.

33. Grasso, D.; Fasone, S.; La Rosa, C. A calorimetric study of the different thermal behaviour of DNA in the isotropic and liquid-crystalline states. Liq. Cryst. 1991, 9, 299-305.

34. Yasar, S.; Podgornik, R.; Valle-Orero, J.; Johnson, M. R.; Parsegian, V. A. Continuity of states between the cholesteric $\rightarrow$ line hexatic transition and the condensation transition in DNA solutions. Sci. Rep. 2014, 4, 1-10, doi:10.1038/srep06877.

35. Zanchetta, G.; Nakata, M.; Buscaglia, M.; Bellini, T.; Clark, N. A. Phase separation and liquid crystallization of complementary sequences in mixtures of nanoDNA oligomers. Proc. Natl. Acad. Sci. U. S. A. 2008, 105, 1111-1117.

36. Fraccia, T. P.; Smith, G. P.; Zanchetta, G.; Paraboschi, E.; Yi, Y.; Walba, D. M.; Dieci, G.; Clark, N. A.; Bellini, T. Abiotic ligation of DNA oligomers templated by their liquid crystal ordering. Nat. Commun. 2015, 6, doi:10.1038/ncomms7424.

37. Fraccia, T. P.; Zanchetta, G.; Rimoldi, V.; Clark, N. A.; Bellini, T. Evidence of Liquid CrystalAssisted Abiotic Ligation of Nucleic Acids. Orig. Life Evol. Biosph. 2015, 45, doi:10.1007/s11084015-9438-1.

38. Todisco, M.; Fraccia, T. Pietro; Smith, G. P.; Zanchetta, G.; Colombo, D.; Bethge, L.; Klussmann, S.; Paraboschi, E.; Asselta, R.; Clark, N. A.; Bellini, T. Liquid Crystal Templated Abiotic Ligation of RNAOligomers. 2018, under subm.

39. Drsata, T.; Pérez, A.; Orozco, M.; Morozov, A. V.; Soner, J.; Lankas, F. Structure, stiffness and substates of the dickerson-drew dodecamer. J. Chem. Theory Comput. 2013, 9, 707-721.

40. Rossi, M.; Zanchetta, G.; Klussmann, S.; Clark, N. a.; Bellini, T. Propagation of chirality in mixtures of natural and enantiomeric dna oligomers. Phys. Rev. Lett. 2013, 110, 107801, doi:10.1103/PhysRevLett.110.107801. 
41. Zanchetta, G.; Bellini, T.; Nakata, M.; Clark, N. A. Physical polymerization and liquid crystallization of RNA oligomers. J. Am. Chem. Soc. 2008, 130, 12864-12865, doi:10.1021/ja804718c.

42. Strey, H.; Parsegian, V.; Podgornik, R. Equation of State for DNA Liquid Crystals: Fluctuation Enhanced Electrostatic Double Layer Repulsion. Phys. Rev. Lett. 1997, 78, 895-898.

43. Bloomfield, V. a DNA condensation by multivalent cations. Biopolymers 1997, 44, 269-282.

44. Wong, G. C. L.; Pollack, L. Electrostatics of strongly charged biological polymers: ion-mediated interactions and self-organization in nucleic acids and proteins. Annu. Rev. Phys. Chem. 2010, 61, 171-189, doi:10.1146/annurev.physchem.58.032806.104436.

45. Raspaud, E.; Durand, D.; Livolant, F. Interhelical spacing in liquid crystalline spermine and spermidine-DNA precipitates. Biophys. J. 2005, 88, 392-403, doi:10.1529/biophysj.104.040113.

46. Burak, Y.; Ariel, G.; Andelman, D. Onset of DNA aggregation in presence of monovalent and multivalent counterions. Biophys. J. 2003, 85, 2100-2110, doi:10.1016/S0006-3495(03)74638-4.

47. Arrighi, F. E.; Mandel, M.; Bergendahl, J.; Hsu, T. C. Buoyant densities of DNA of mammals. Biochem. Genet. 1970, 4, 367-376, doi:10.1007/BF00485753.

48. J., T. N.; M., L. S.; A., R. Dynamic coupling between DNA and its primary hydration shell studied by brillouin scattering. Biopolymers 27, 1655-1671, doi:10.1002/bip.360271010.

49. Feig, M.; Montgomery Pettitt, B. A molecular simulation picture of DNA hydration around A- And B-DNA. Biopolymers 1998, 48, 199-209, doi:10.1002/(SICI)1097-0282(1998)48:4<199::AIDBIP2>3.0.CO;2-5.

50. Parsegian, V. A.; Rand, R. P.; Rau, D. C. Osmotic stress, crowding, preferential hydration, and binding: A comparison of perspectives. Proc. Natl. Acad. Sci. 2000, 97, 3987-3992, doi:10.1073/pnas.97.8.3987.

51. Linegar, K. L.; Adeniran, A. E.; Kostko, A. F.; Anisimov, M. A. Hydrodynamic radius of polyethylene glycol in solution obtained by dynamic light scattering. Colloid J. 2010, 72, 279-281, doi:10.1134/S1061933X10020195.

52. Gurnev, P. A.; Stanley, C. B.; Aksoyoglu, M. A.; Hong, K.; Parsegian, V. A.; Bezrukov, S. M. Poly(ethylene glycol)s in Semidilute Regime: Radius of Gyration in the Bulk and Partitioning into a Nanopore. Macromolecules 2017, 50, 2477-2483, doi:10.1021/acs.macromol.6b02571.

53. Rubinson, K. A.; Krueger, S. Poly(ethylene glycol)s 2000-8000 in water may be planar: A smallangle neutron scattering (SANS) structure study. Polymer (Guildf). 2009, 50, 4852-4858, doi:10.1016/j.polymer.2009.08.023.

54. Kranendonk, W. G. T.; Frenkel, D. Thermodynamic properties of binary hard sphere mixtures. Mol. Phys. 1991, 72, 715-733, doi:10.1080/00268979100100521.

55. Chen, A. A.; Garcia, A. E. High-resolution reversible folding of hyperstable RNA tetraloops using molecular dynamics simulations. Proc. Natl. Acad. Sci. 2013, 110, 16820-16825, doi:10.1073/pnas.1309392110.

56. Lucchetti, L.; Fraccia, T. P.; Ciciulla, F.; Bellini, T. Non-linear optical measurement of the twist elastic constant in thermotropic and DNA lyotropic chiral nematics. Sci. Rep. 2017, 7, doi:10.1038/s41598-017-05136-Z.

57. Lucchetti, L.; Fraccia, T. P.; Ciciulla, F.; Simoni, F.; Bellini, T. Giant optical nonlinearity in DNA lyotropic liquid crystals. Opt. Express 2017, 25, 25951, doi:10.1364/OE.25.025951. 